\title{
Streamlined scanning for enhancer elements in Drosophila melanogaster
}

\author{
Roumen Voutev ${ }^{1}$ and Richard S. Mann ${ }^{1}$ \\ ${ }^{1}$ Department of Biochemistry and Molecular Biophysics, Columbia \\ University, New York, NY
}

BioTechniques 60:141-144 (March 2016) doi 10.2144/000114391

Keywords: enhancer; CRM; reporter construct; D. melanogaster

Enhancer elements in most eukaryotic organisms are often positioned at a great distance away from the transcription start site of the gene they regulate. Complex three-dimensional chromatin organization and insulators usually guide and limit the range of an enhancer's regulatory activity to a specific genetic locus. Rigorous testing of an entire genomic locus is often required in order to uncover the complete set of cis-regulatory modules (CRMs) regulating a gene, especially those with complex and dynamic expression patterns. Here we report a fast and efficient method for enhancer element identification by scanning large genomic regions using transgenic reporter genes.

The fruit fly Drosophila melanogaster has long been a key model organism for investigation of enhancer element structure and function. Genome-scale chromatin studies assisted by bioinformatic approaches have described many of the cis-regulatory modules (CRMs) and their architecture throughout the fruit fly genome (reviewed in Reference 1). However, enhancer elements that are active at particular developmental stages might easily be missed using standard approaches. In addition, in many cases multiple shadow enhancers function in a semi-redundant manner, and their combined analysis is required to fully describe a gene's transcriptional regulation. Because enhancers can be far apart from one another and from the transcription start sites of the genes they regulate, we have developed a novel technique to rapidly and efficiently scan large genomic regions.

Venken and colleagues generated valuable genomic libraries, CHORI-322 and $\mathrm{CHORI}-321$, which cover the Drosophila genome and can be used for gene rescue experiments and gene tagging since the backbone contains a selectable marker (mini-white+) and phiC31 attB site for transgenesis (2). While CHORI-321 consists of clones carrying large genomic fragments (average size $80 \mathrm{~kb}$ ), $\mathrm{CHORI}-322$ was designed to carry only $\sim 20 \mathrm{~kb}$ fragments (available at BACPAC Resource Center, Oakland, CA). Because of their relatively small size, CHORI-322 clones can be efficiently inserted into the genome using the phiC31 system (2). Since the CHORI-322 library virtually tiles the entire genome, we reasoned that even a large gene locus of $\sim 200 \mathrm{~kb}$ (from one insulator to another) could be scanned with $10 \mathrm{CHORI}-322$ clones.

To create reporter constructs from the $\mathrm{CHORI}-322$ clones, all that is required is insertion at a feasible location along the DNA fragment of a reporter gene containing its own minimal promoter and sequences for transcriptional termination. This is quickly achievable by using a unique restriction site in each clone (there are many available in any $20 \mathrm{~kb}$ fragment) to open the vector and insert a promoter-reporter-3'-terminator PCR product through Gibson Assembly (3). Each set of primers in this case would contain 25 bp overhangs that are complementary to the corresponding clone sequence flanking the chosen restriction enzyme site (Figure 1A). To avoid the possibility that a putative enhancer element would be destroyed at the chosen restriction site location, an overlapping BAC clone could be used to cover the site of the reporter insertion in another construct. In addition, two different reporter cassettes could be inserted in opposite ends of a BAC in order to be able to compare expression of both in cases where secondary DNA structure or repressive elements are predicted or suspected in a particular genomic region.

To test this strategy, we chose the 3' region of the gene teashirt (tsh), which spans $\sim 60 \mathrm{~kb}$ to the next downstream gene (Figure $1 \mathrm{~B})$ and contains numerous non-coding RNAs. There are no predicted strong insulators (as defined by modENCODE; www.modencode.org) in this region that would prevent a putative enhancer from activating the inserted reporter gene and generating an expression pattern. This $60 \mathrm{~kb}$ region could be covered with only 3 CHORI-322 clones: 176G09, 161P06, and 74E24 (Figure 1B). These clones were digested with enzymes SanDI, BsiWl, and Ascl, respectively, and purified with the QIAquick PCR Purification Kit (\#28106; Qiagen, Valencia, CA). PCR was used to amplify a cassette carrying the Drosophila synthetic minimal promoter (4) driving tdTomato (5) followed by the SV40 late polyA 3' terminator (6) (synthP-tdTomato-SV4O), and to insert each reporter cassette, flanked by clone-specific overhangs, into the corresponding clones using Gibson Assembly (Figure 1A). The resulting mix was transformed by electroporation into TransforMax EPI300 E. coli cells (\#EC300110; Epicenter Biotechnologies, Madison, WI). Each transformation generated $\sim 30$ colony-forming units (CFUs). We grew three colonies per construct in standard LB media and induced each of them to high-copy number using CopyControl solution (\#CCIS125; Epicenter Biotechnologies). We extracted DNA corresponding to each colony via the QIAprep

\section{METHOD SUMMARY}

We developed a method for quick discovery of novel cis-regulatory modules (CRMs) by generating enhancer-reporter constructs that span vast genomic regions in the fruit fly Drosophila melanogaster. Our method introduces a small promoter-reporter-3'-terminator cassette into phiC31 system-compatible CHORI-322 clones using Gibson Assembly. 
A

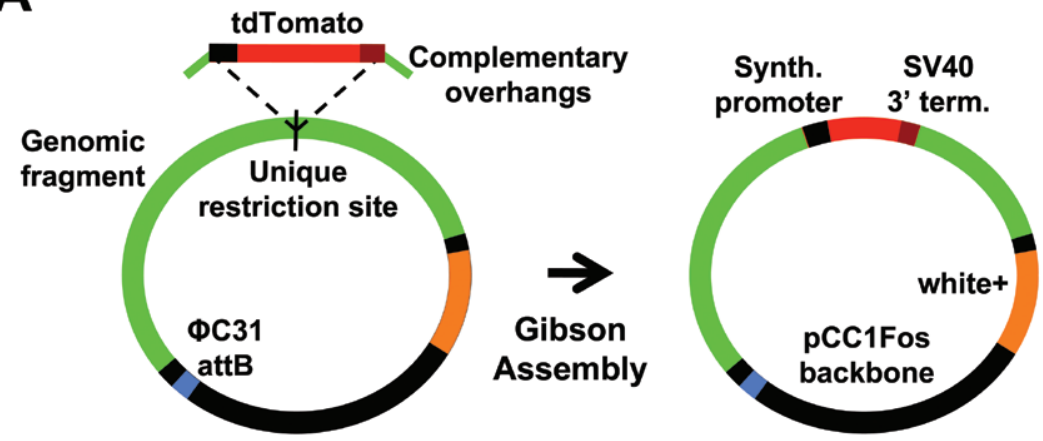

B

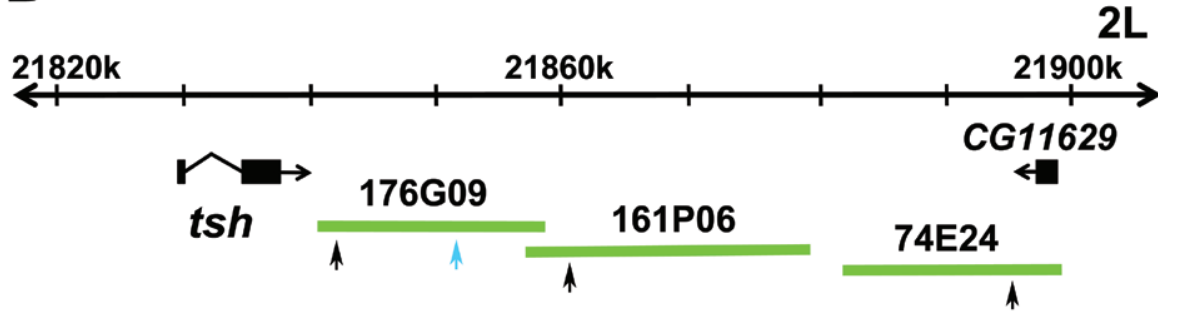

Figure 1. Efficient generation of enhancer-reporter constructs using Drosophila melanogaster BAC clones. (A) Schematic representation of the conversion of any CHORI-322 clone into a reporter construct through Gibson Assembly. The following oligonucleotides were used for PCR amplification of the synthP-tdTomato-SV4O cassette: 5'-cgcactgtgaaaggggtgaggcggtacagtATCGAGCGCAGCGGTATAAAAGGGCGCGGG-3' and 5'-aatattttacaattggtgcceccgccaactAAAAAACCTCCCACACCTCCCCCTGAACCT-3' in the case of clone 176G09; 5'-gtttatttatataagtgaagaatccgtacgATCGAGCGCAGCGGTATAAAAGGGCGCGGG-3' and 5'-atcataatcggaattcaaataattaagatCAAAAAACCTCCCACACCTCCCCCTGAACCT-3" in the case of clone 161P06; 5'-caagccaaaaatatggaatagattgggaaATCGAGCGCAGCGGTATAAAAGGGCGCGGG-3' and 5'-gattgctgctctttgtttttgtgtgctggCAAAAAACCTCCCACACCTCCCCCTGAACCT-3' in the case of clone 74E24; lower case letters represent complementary overhangs. (B) Schematic representation of the tsh 3 ' genomic region on chromosome $2 \mathrm{~L}$ and the $\mathrm{CHORI}-322$ clones used in this study; black arrows represent the sites of reporter cassette insertion; blue arrow represents the relative location of a previously described tsh embryonic cis-regulatory module (CRM) (9).

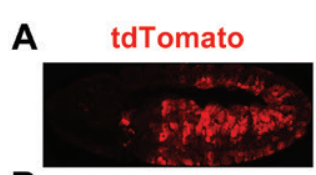

B

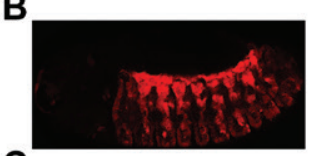

C

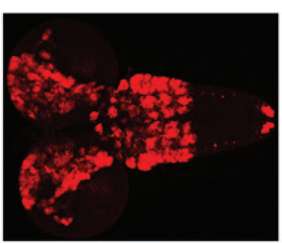

D

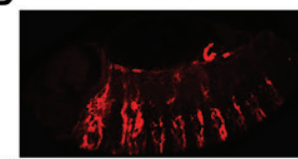

E
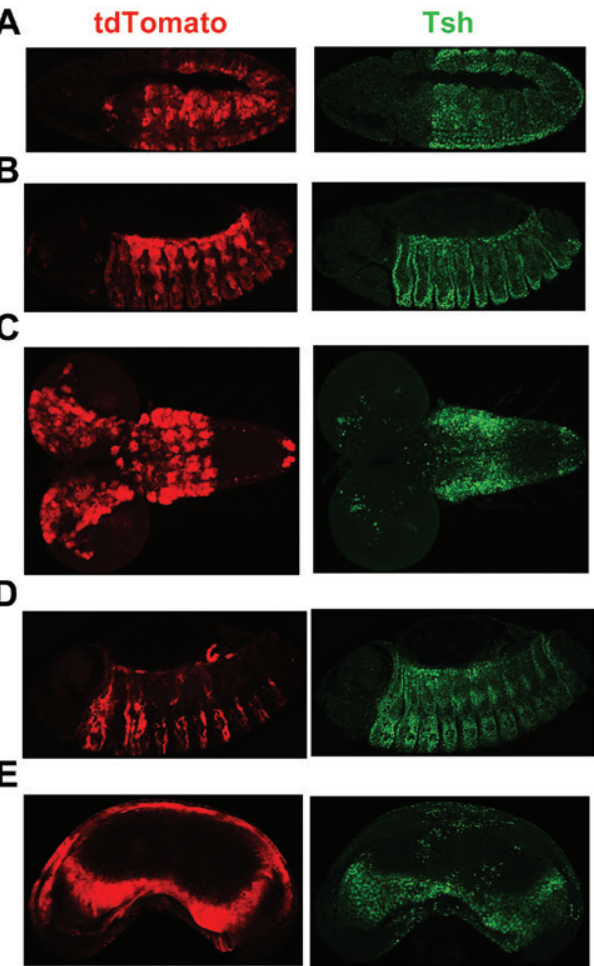

$\mathbf{F}$
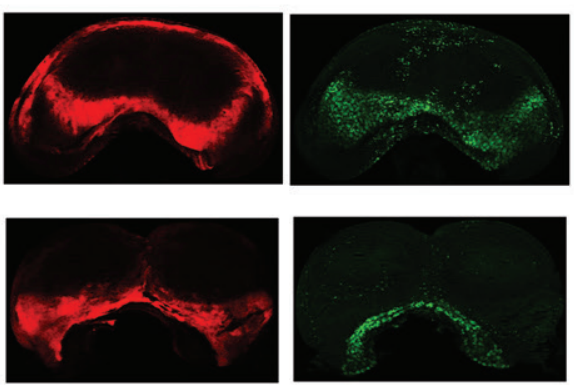

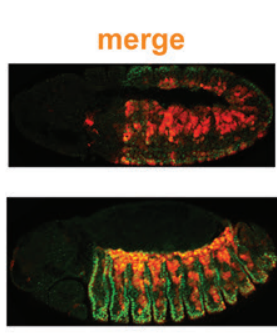

G

tdTomato

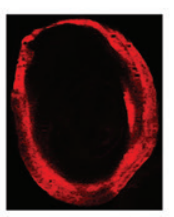

H
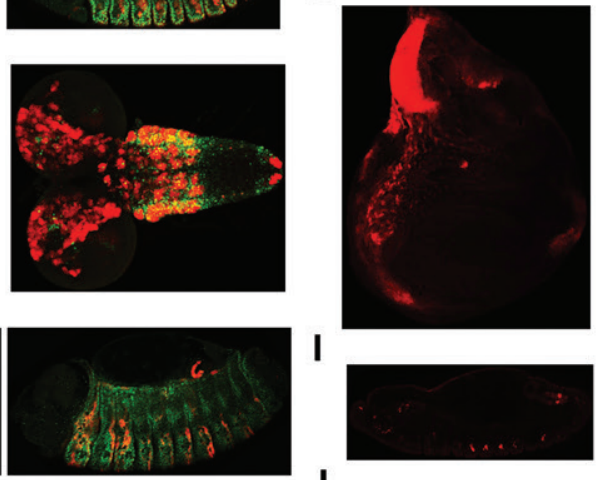

I
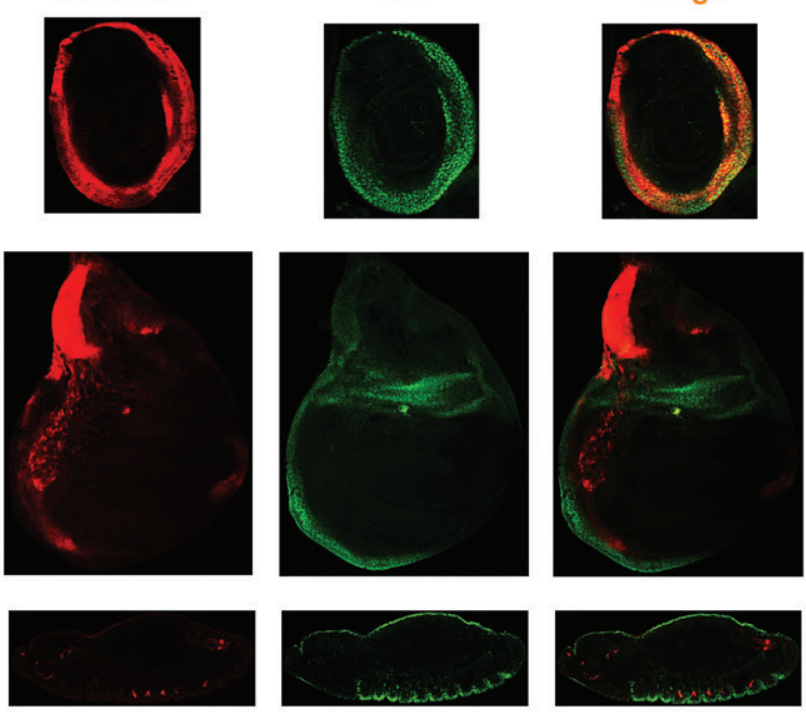

J
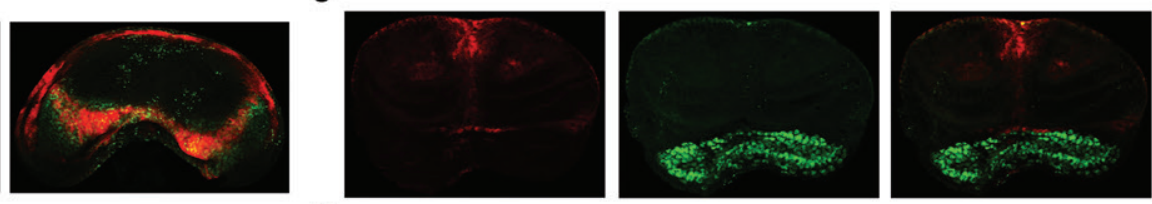

K
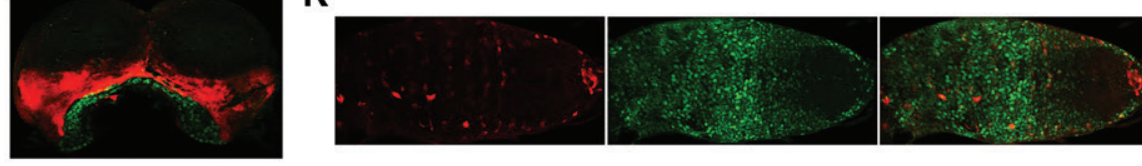

Figure 2. Expression pattern of the generated reporter constructs. Comparison between the expression of tdTomato (red) with tsh (green) in a 176G09-tdTomato stage 11 embryo (A), a 176G09-tdTomato stage 14 embryo (B), a 176G09-tdTomato larval brain (C), a 161P06-tdTomato stage 14 embryo (D), a 161P06tdTomato L3 larval female genital imaginal disc (E), a 161P06-tdTomato L3 larval male genital imaginal disc (F), a 161P06-tdTomato L3 larval leg imaginal disc (G), a 161P06-tdTomato L3 larval wing imaginal disc (H), a 74E24-tdTomato stage 14 embryo (I), a 74E24-tdTomato L3 larval male genital imaginal disc (J), and a 74E24-tdTomato L3 larval ventral nerve cord (VNC) (K). Note that tdTomato is non-nuclear and Teashirt is nuclear. Immunostaining was performed with goat anti-tdTomato antibody (\#LS-C340696; LifeSpan BioSciences, Seattle, WA) and guinea pig anti-Teashirt antibody (gift from Gary Struhl). Corresponding donkey Alexa-Fluor-conjugated secondary antibodies [donkey anti-goat Alexa Fluor 555 (ThermoFisher Scientific, Waltham, MA) and donkey anti-guinea pig Alexa Fluor 488 (Jackson ImmunoResearch Laboratories, West Grove, PA)] were used to visualize the primary antibody staining. Anterior is left; posterior is right. 
Spin Miniprep Kit (\#27106; Qiagen) and verified each of the constructs by sequencing the synthP-tdTomato-SV4O minigene and insertion site. Next, we extracted sufficient amounts of DNA for injection ( $250 \mathrm{ng} / \mu \mathrm{l})$ with the Qiagen Plasmid Midi Kit (\#12143; Qiagen) and generated transgenic flies carrying each of the constructs using the phiC31 system $(7,8)$ and the attP2 insertion site $(7)$.

We analyzed the expression pattern of the generated reporter constructs in fly imaginal discs, embryos, and larval nervous system. Given the size of each construct, multiple CRMs may contribute to the reporter expression in each case. Clone 176 G09 contains a previously described Hox-dependent tsh embryonic enhancer (9), and the transgene made with this clone recapitulated tsh's embryonic expression pattern, verifying that the reporter cassette is capable of recruiting, at the very least, this tsh embryonic CRM (Figure 2, A and B), which is located $\sim 10 \mathrm{~kb}$ downstream of the reporter cassette insertion site (Figure 1B). In addition, clone 176G09 drove expression in the larval brain that co-localizes with the tsh expression in the ventral nerve cord (VNC) but diverges from the tsh expression in the lobes of the brain (Figure 2C). This suggests that the region covered by 176 G09 contains at least one tsh VNC enhancer element. Clone 176G09 did not drive expression in third instar larval imaginal discs.

Clone 161P06 drove expression in a tsh-like pattern in stage 14 embryos (Figure 2D), which argues that this region contains additional embryonic tsh CRMs. This clone also drove expression in multiple imaginal discs. Some patterns coincided nicely with the tsh expression, while in other cases, the 161P06-tdTomato expression deviated from that of $t s h$. For example, in female genital discs 161P06-tdTomato matches almost completely the tsh expression pattern (Figure $2 \mathrm{E})$, while in male genital discs the expression driven by 161P06 is adjacent to that of tsh (Figure 2F). Most likely this region contains one or more legitimate female genital disc CRMs, while the male genital CRM is either interrupted or several CRMs are required for the correct expression pattern of tsh in male genital imaginal discs and some of them are positioned in different genomic regions (e.g., 5 of $t s h)$. The reporter expression driven by clone 161P06 also overlapped well with the tsh expression pattern in leg imaginal discs, suggesting the presence of one or more tsh leg CRMs in this region (Figure 2G). In contrast to the leg disc, 161P06-tdTomato drove expression in the proximal wing disc that overlapped with tsh expression only in a few regions (Figure $2 \mathrm{H}$ ). The expression pattern in the haltere disc was analogous (data not shown), suggesting that there are other tsh wing and haltere CRMs outside of $161 P 06$.

Clone 74E24 generated some reporter expression in stage 14 embryos (Figure 2l), in male genital imaginal discs (Figure 2J), and in the larval nervous system (Figure 2K), none of which co-localized with the tsh expression, suggesting that this region contains regulatory elements unrelated to tsh. None of the generated reporter constructs drove expression in the mouth and eye-antennal imaginal discs (although tsh is expressed in the anterior eye disc).

The method described here allows for rapid and efficient scanning of any Drosophila genomic region as a first step toward identifying enhancer elements of a gene of interest at any developmental stage and tissue. It will complement other genomewide tiling projects (10-12) and computa-

\section{The BMG LABTECH All Stars}

\section{Innovative, high-performance microplate readers for all assay needs}

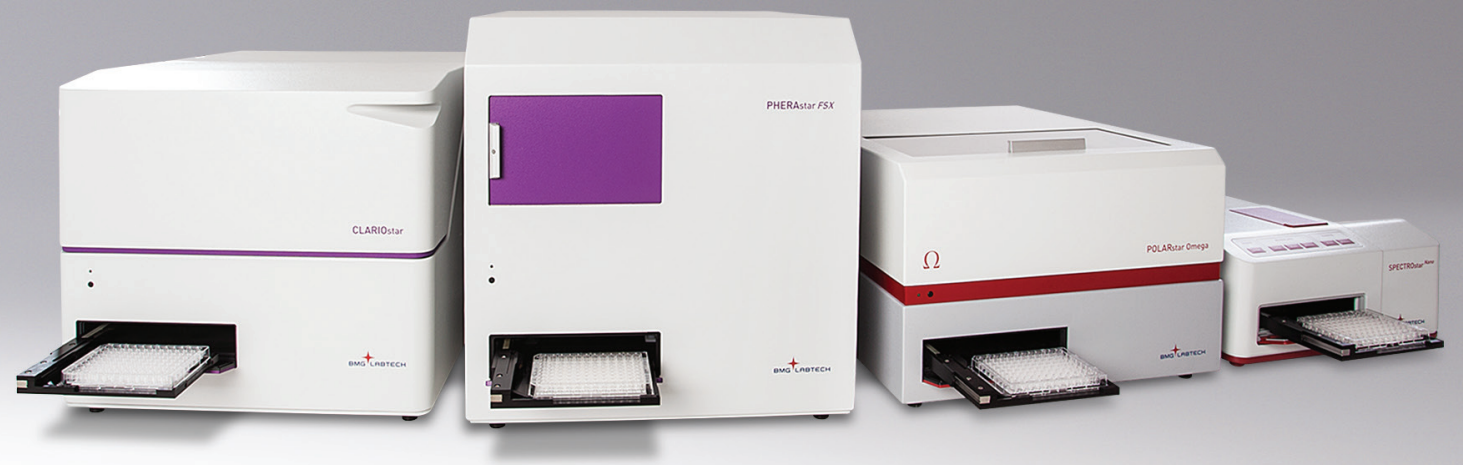

\section{CLARIOstar ${ }^{\circledR}$ \\ The most sensitive monochromator-based microplate reader.}

\begin{abstract}
Omega series
Upgradable filter-based microplate readers for any life science application.
\end{abstract}

SPECTROstar ${ }^{\circledR}$ Nano

Absorbance reader with ultra-fast detection of UV/Vis absorbance spectra. 
tional approaches (reviewed in References 13 and 14), thus significantly improving the identification and verification of putative CRMs.

\section{Author contributions}

R.V. conceived this work, performed the experiments, and wrote the article. R.S.M. edited the article text and supported this study.

\section{Acknowledgments}

Thanks to Jonathan Enriquez and Laura Quintana Rio for technical assistance. R.V. is a Leukemia and Lymphoma Society fellow. This work was supported by NIH grant GM058575 awarded to R.S.M. This paper is subject to the $\mathrm{NIH}$ Public Access Policy.

\section{Competing interests}

The authors declare no competing interests.

\section{References}

1. Lelli, K.M., M. Slattery, and R.S. Mann. 2012. Disentangling the many layers of eukaryotic transcriptional regulation. Annu. Rev. Genet. 46:43-68.
2. Venken, K.J., J.W. Carlson, K.L. Schulze, H. Pan, Y. He, R. Spokony, K.H. Wan, M. Koriabine, et al. 2009. Versatile P[acman] BAC libraries for transgenesis studies in Drosophila melanogaster. Nat. Methods 6:431-434.

3. Gibson, D.G., L. Young, R.Y. Chuang, J.C. Venter, C.A. Hutchison 3rd, and H.O. Smith. 2009. Enzymatic assembly of DNA molecules up to several hundred kilobases. Nat. Methods 6:343-345.

4. Pfeiffer, B.D., A. Jenett, A.S. Hammonds, T.T. Ngo, S. Misra, C. Murphy, A. Scully, J.W. Carlson, et al. 2008. Tools for neuroanatomy and neurogenetics in Drosophila. Proc. Natl. Acad. Sci. USA 105:9715-9720.

5. Shaner, N.C., R.E. Campbell, P.A. Steinbach, B.N. Giepmans, A.E. Palmer, and R.Y. Tsien. 2004. Improved monomeric red, orange and yellow fluorescent proteins derived from Discosoma sp. red fluorescent protein. Nat. Biotechnol. 22:15671572.

6. Fitzgerald, M. and T. Shenk. 1981. The sequence 5'-AAUAAA-3'forms parts of the recognition site for polyadenylation of late SV40 mRNAs. Cell 24:251260.

7. Groth, A.C., M. Fish, R. Nusse, and M.P. Calos. 2004. Construction of transgenic Drosophila by using the site-specific integrase from phage phiC31. Genetics 166:1775-1782.

8. Bischof, J., R.K. Maeda, M. Hediger, F. Karch, and K. Basler. 2007. An optimized transgenesis system for Drosophila using germ-line-specific phiC31 integrases. Proc. Natl. Acad. Sci. USA 104:3312-3317.

9. McCormick, A., N. Core, S. Kerridge, and M.P. Scott. 1995. Homeotic response elements are tightly linked to tissue-specific elements in a transcriptional enhancer of the teashirt gene. Development 121:2799-2812.

10. Kvon, E.Z., T. Kazmar, G. Stampfel, J.O. Yanez-Cuna, M. Pagani, K. Schernhuber, B.J. Dickson, and A. Stark. 2014. Genome-scale functional characterization of Drosophila developmental enhancers in vivo. Nature 512:91-95.

11. Jory, A., C. Estella, M.W. Giorgianni, M. Slattery, T.R. Laverty, G.M. Rubin, and R.S. Mann. 2012. A survey of 6,300 genomic fragments for cis-regulatory activity in the imaginal discs of Drosophila melanogaster. Cell Rep. 2:1014-1024.

12. Jenett, A., G.M. Rubin, T.T. Ngo, D. Shepherd, C. Murphy, H. Dionne, B.D. Pfeiffer, A. Cavallaro, et al. 2012. A GAL4-driver line resource for Drosophila neurobiology. Cell Rep. 2:991-1001.

13. Shlyueva, D., G. Stampfel, and A. Stark. 2014. Transcriptional enhancers: from properties to genome-wide predictions. Nat. Rev. Genet. 15:272-286.

14. Suryamohan, K. and M.S. Halfon. 2015. Identifying transcriptional cis-regulatory modules in animal genomes. Wiley Interdiscip Rev Dev Biol. 4:59-84.

Received 16 September 2015; accepted 09 December 2015.

Address correspondence to Richard S. Mann or Roumen Voutev, Department of Biochemistry and Molecular Biophysics, Columbia University, 701 W168 th St. HHSC 1104, New York, NY 10032. E-mail: rsmann10@gmail.com or roumen.voutev@gmail.com

To purchase reprints of this article, contact: biotechniques@fosterprinting.com

\section{RNAzol ${ }^{\circledR}$ RT Column Kit}

Universal RNA isolation kit from Molecular Research Center The market leader providing the best RNA isolation methods for over 25 years

Fast and dependable isolation

Highest yield and purity

No DNase treatment necessary
Process small or large samples

Up to $\mathbf{3 0 0}$ mg RNA per column

Variety of solid or liquid samples

\section{Specialized protocols for the isolation of:}

Total RNA containing all cellular RNA Large RNA containing mRNA, rRNA and non-coding RNA Small RNA containing micro RNA

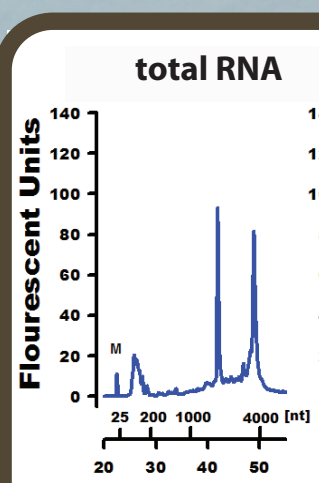

large RNA

small RNA
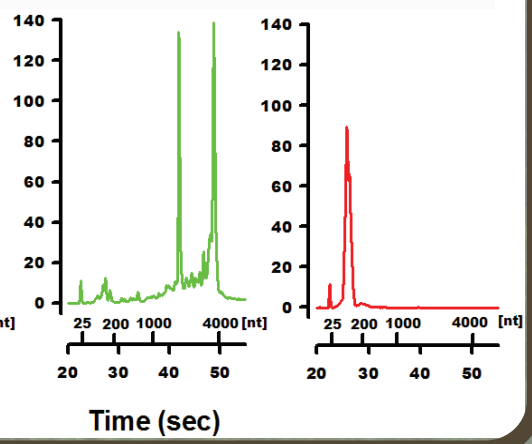